\title{
RELAÇÃO ENTRE ANSIEDADE, DEPRESSÃO E DESESPERANÇA ENTRE GRUPOS DE IDOSOS
}

\author{
Katya Luciane de Oliveira* \\ Acácia Aparecida Angeli dos Santos \\ Mirian Cruvinel \\ Anita Liberalesso Néri ${ }^{\circledast 2}$
}

\begin{abstract}
RESUMO. O envelhecimento é uma etapa da vida que permanece pouco conhecida e estudada, se comparada a outras fases do desenvolvimento humano. Nesta pesquisa buscou-se explorar a relação entre ansiedade, depressão e desesperança entre grupos de idosos. Participaram deste estudo 79 idosos provenientes de centro do terceira idade de um posto de retirada de medicamentos e de uma instituição asilar. Utilizou-se um questionário para os dados de caracterização dos idosos, bem como as escalas Beck para mensurar sintomas de ansiedade, depressão e desesperança. Os resultados evidenciaram relação estatisticamente significativa entre ansiedade, depressão e desesperança nos idosos. O grupo de asilares apresentou uma maior incidência de sintomas ansiosos, depressivos e desesperançosos em relação aos outros dois grupos.
\end{abstract}

Palavras-chave: psicologia do envelhecimento, velho, saúde mental.

\section{RELATIONS BETWEEN ANXIETY, DEPRESSION AND HOPELESSNESS AMONG ELDERLY GROUPS}

\begin{abstract}
Aging is a life stage that remains hardly ever studied and, consequently, unknown if compared to other human development phases. This research investigates the relation between anxiety, depression and hopelessness among elderly groups. 79 elderly people, from three different units, such as a 'third-age-center', a center of distribution of medicine, and a nursing home, took part in the study. The material used in the study was a questionnaire for data characterization, as well as, Beck's scales that was used to evaluate anxiety, depression and hopelessness. The results indicated a significant association between anxiety, depression and hopeless in elderly people. The elderly group living in a nursing home showed more incidence of anxiety, depression and hopelessness symptoms when compared to the two others groups.
\end{abstract}

Key words: Aging psychology, elderly, mental health.

\section{RELACIÓN ENTRE ANSIEDAD, DEPRESIÓN Y DESESPERANZA ENTRE GRUPOS DE ANCIANOS}

RESUMEN. El envejecimiento es una etapa de la vida que permanece poco conocida o estudiada, al ser comparada a otras fases del desarrollo humano. Esta investigación buscó explorar la relación entre ansiedad, depresión y desesperanza entre grupos de ancianos. Participaron en este estudio 79 adultos mayores provenientes de tres sitios diferentes: un centro de tercera edad, un puesto de retirada de medicinas y un asilo. Un cuestionario para los datos de caracterización de los ancianos fue utilizado, así como las escalas Beck para mensurar síntomas de ansiedad, depresión y desesperanza. Los resultados dejaron en evidencia relación estadísticamente significativa entre dichos síntomas en los adultos mayores. El grupo proveniente del asilo presentó mayor incidencia de manifestaciones en relación con los demás grupos.

Palabras-clave: psicología del envejecimiento, anciano, salud mental.

* Psicóloga. Mestre. Doutoranda no Programa de Pós-Graduação em Psicologia, Desenvolvimento Humano e Educação pela Faculdade de Educação da Universidade Estadual de Campinas e docente do curso de Psicologia da Universidade São Francisco.

\# Psicóloga. Doutora em educação pela Universidade de São Paulo e docente do curso de Psicologia e do Programa de Pósgraduação Stricto sensu em Psicologia da Universidade São Francisco-Itatiba/SP. Bolsista Produtividade do CNPq.

Il Psicóloga. Doutoranda em Psicologia, Desenvolvimento Humano e Educação pela Faculdade de Educação da Universidade Estadual de Campinas.

x Doutora. Docente do Programa de Pós-Graduação da Universidade Estadual de Campinas. 
A psicologia do envelhecimento é uma área de investigação relativamente recente, que teve seu início com os avanços obtidos pela gerontologia. As primeiras investigações sobre a velhice, segundo Birren e Schroots (2001), datam de 1835, quando um cientista belga publicou um livro no qual abordava as diferenças entre aspectos físicos e comportamentais de indivíduos de acordo com a idade. Nesta obra os autores evidenciaram que havia um período no qual a memória, a imaginação e o julgamento apresentavam uma maturidade e em algumas pessoas poderia apresentar um declínio. Tratava-se apenas de mais um estágio do desenvolvimento.

O envelhecimento inicia-se imediatamente após a fecundação, visto que muitas células envelhecem, morrem e são substituídas antes mesmo de nascer. Estudiosos do tema têm constatado que envelhecer é um acontecimento natural que compõe o desenvolvimento normal e integral de qualquer pessoa, sendo produto de um processo dinâmico de uma vida na qual o indivíduo se modifica incessantemente. Assim, é um processo biológico universal, pelo qual o ser humano é necessariamente levado à velhice (Albuquerque, 1994; Fraiman, 1995; Santos, 1994).

$\mathrm{O}$ indivíduo é concomitantemente produtor e produto de uma sociedade e de sua cultura. Portanto, por estar em uma fase marcada por grandes transformações psicológicas, físicas e sociais, o idoso deve ter consciência de si enquanto ser finito, que inevitavelmente envelhece. Somente assim poderá vivenciar sua velhice com tranqüilidade (Neri, 1995).

Para Bee e Mitchell (1984), a maioria dos idosos experimenta uma significativa alteração em seu cotidiano com o início da aposentadoria e papéis profissionais. Muitos apresentam modificações orgânicas como alterações cognitivas e perceptivas, bem como transformações importantes nos relacionamentos sociais.

Antonucci (2001) considera que as relações sociais nessa fase são permeadas mais intensamente por influências de fatores psicológicos, físicos e da saúde mental. Todavia, quando um idoso perde a capacidade de se recordar dos fatos, alguns relacionamentos, inclusive com familiares, podem ficar abalados, ao passo que muitas pessoas que convivem com o idoso não compreendem as mudanças que ocorrem na vida da pessoa.

Nesse sentido, Haddad $(1986,1993)$ defende que o envelhecimento imprime aos indivíduos alterações naturais. No entanto, assinala que há diferença entre o envelhecimento fisiológico, que ocorre em razão da idade cronológica, e o patológico, que pode até afetar pessoas não idosas, por depender principalmente de como a pessoa lida com a realidade e seu mundo.

Para alguns idosos, tais alterações se caracterizam pelo que Bee e Mitchell (1984) chamam de desligamento, uma vez que, nessa fase, alguns idosos tendem a se desligar de suas obrigações e de seus papéis sociais e profissionais. Por outro lado, existem aqueles que vivenciam a velhice de uma forma mais positiva, adotam uma postura mais ativa, com freqüentes atividades físicas e de lazer. Certamente, os indivíduos que experienciam a velhice de maneira negativa, que se afastam das atividades diárias e dos relacionamentos sociais, apresentam uma maior probabilidade de também vivenciarem emoções negativas.

Lawton (2001) aponta que para se ter uma boa qualidade de vida no envelhecer é necessário o desenvolvimento de pesquisas que enfatizem não somente aspectos físicos e sociais, mas principalmente aqueles relacionados à saúde emocional. Neri e Nogueira (1994) salientam que os aspectos socioculturais e condições econômicas também desempenham papel no processo de envelhecer com qualidade; ressaltam ainda que somente com o desenvolvimento da área, novas alternativas surgirão para um envelhecer saudável.

\section{ANSIEDADE, DEPRESSÃO E DESESPERANÇA EM IDOSOS}

Muitos idosos nessa etapa da vida são acometidos por distúrbios mentais. A pesquisa de Maia, Durante e Ramos (2004) evidenciou a prevalência desses distúrbios em 29,3\% dos 327 idosos investigados. Em razão da alta taxa de problemas psiquiátricos ou emocionais em idosos, o número de pessoas que procuram serviços de atendimento em saúde mental cresce a cada ano.

Avaliar quais os motivos que levam o idoso a procurar o serviço médico foi o foco do estudo de Almeida (1999). Os dados levantados sugerem que o transtorno do humor foi o problema psiquiátrico mais freqüente, sendo muito comum entre as mulheres. Também foram encontrados diagnósticos de síndrome demencial, transtornos ansiosos, esquizofreniformes, alcoolismo e abuso de sedativos. Contudo, Gazalle, Hallal e Lima (2004) apontam que problemas psicológicos tais como ansiedade, depressão e desesperança em idosos são pouco investigados pelos médicos ou outros profissionais no contexto clínico, embora sejam comuns como queixas clínicas.

No caso da ansiedade, são frequientes sintomas como insônia, tensão, angústia, irritabilidade, 
dificuldade de concentração, bem como sintomas físicos como taquicardia, tontura, cefaléia, dores musculares, formigamento, suor. Para o diagnóstico de uma síndrome ansiosa é importante verificar a intensidade dos sintomas e seu impacto na vida do indivíduo (Dalgalarrondo, 2000).

A ansiedade ocorre diante de uma visão catastrófica dos eventos, anunciando que algo perigoso e ameaçador pode acontecer. Para Skinner e Vaughan (1985), a ansiedade nos idosos está relacionada às limitações vivenciadas na velhice e, na maioria das vezes, interpretadas como ameaçadoras. As pessoas com altos níveis de ansiedade apresentam uma tendência de antecipar sua inabilidade e questionar suas habilidades intelectuais. Essas percepções negativas interferem na atenção seletiva, na codificação de informações na memória, bloqueando a compreensão e o raciocínio (Coes, 1991), o que nessa fase da vida poderia ser a diferença entre uma saúde mental boa ou comprometida.

Byrne (2002) destaca que são freqüentes sintomas de ansiedade em idosos, e na maioria das vezes, a ansiedade vem associada a transtornos depressivos e a doenças físicas. Todavia, há poucas investigações a respeito da prevalência de ansiedade na população acima de 65 anos.

A ansiedade em idosos com mais de 80 anos foi tema da pesquisa de Xavier e cols. (2001), na qual constatou que $10,6 \%$ dos 77 idosos participantes apresentavam transtorno de ansiedade generalizada. Os elevados índices de ansiedade estavam associados com sintomas depressivos, transtorno depressivo maior e depressão menor. Os autores ressaltam a importância da atitude do médico diante do idoso, devendo considerar a presença de comorbidades entre ansiedade e humor depressivo.

Em um estudo epidemiológico com 18571 pessoas, Regier e cols. (1988) verificaram que 5,5\% dos idosos com mais de 65 anos de idade apresentavam transtorno ansioso, excluindo os casos mais comuns de ansiedade generalizada. Esse índice aumenta para $15 \%$ quando são incluídos casos de ansiedade generalizada (Katona, Manela \& Livingston, 1996). No estudo de Almeida (1999) com 398 idosos foi encontrada uma prevalência de $15,4 \%$ de idosos com transtorno de ansiedade.

No que tange à depressão, é importante destacar que ela tem sido considerada como um dos transtornos que mais afetam o idoso. Seu diagnóstico no idoso é ainda mais complexo quando comparado ao diagnóstico em outras faixas etárias, pois, como ressaltam Piccoloto, Wainer, Benvegnú e Juruena (2001), o próprio processo de envelhecimento apresenta algumas características semelhantes aos sintomas depressivos, o que conduz à confusão no diagnóstico.

Conforme descrito no Manual de Diagnóstico e Estatístico de Transtornos Mentais - DSM-IV (1994), para o diagnóstico de um Episódio Depressivo Maior é necessário que o indivíduo apresente, durante um período de pelo menos duas semanas, cinco ou mais dos sintomas listados a seguir: humor deprimido na maior parte do dia e em quase todos os dias; falta de interesse ou prazer em todas ou quase todas as atividades na maior parte do dia; perda ou ganho de peso sem estar de dieta; insônia ou hipersonia quase todos os dias; agitação ou retardo psicomotor; fadiga ou perda de energia; sentimento de inutilidade ou de culpa; indecisão e dificuldade de concentração; pensamentos de morte ou tentativas de suicídio. Além disso, os sintomas devem causar sofrimento ou prejuízo ao funcionamento do indivíduo e não devem ser conseqüência de uso de substâncias como drogas ou algum tipo de medicamento, nem ocorrer em função de uma condição médica ou de luto.

A depressão envolve uma série de comprometimentos no funcionamento de uma pessoa. Os resultados do estudo de Fleck e cols. (2002) mostraram que a presença de sintomatologia depressiva está associada com pior funcionamento social e qualidade de vida, bem como a uma maior utilização dos recursos de saúde. Os indivíduos com maior intensidade de sintomas depressivos avaliaram sua saúde como pior e estão menos satisfeitos com ela do que aqueles com menor intensidade de sintomas depressivos.

Muitos estudiosos têm se preocupado em estudar características de idosos que vivem em abrigos ou asilos. Entre eles, Davim, Torres, Dantas e Lima (2004) procuraram conhecer o contexto social, econômico e de saúde de idosos que moram em asilos. Os participantes do estudo foram 76 idosos que viviam em três instituições asilares diferentes, os quais foram submetidos a entrevistas. Os resultados indicaram que, assim como aponta a literatura, a maioria dos idosos é do sexo feminino, possui baixo nível de escolaridade, dificuldades financeiras, relacionamento familiar conflituoso, atividades de lazer limitadas e problemas de saúde.

A autonomia do idoso institucionalizado foi o foco da pesquisa de Garbin e Montewka (2000). Foram entrevistados 306 idosos de uma instituição geriátrica do Estado de São Paulo. Os resultados evidenciaram que quando a instituição asilar oferece condições que favorecem a realização de atividades facilitadoras da autonomia e independência dos idosos, há motivação e poucos sentimentos negativos relacionados à velhice. 
Nesse contexto, percepções negativas e autoderrotistas podem conduzir o idoso a desenvolver sentimentos de desesperança. A desesperança caracteriza-se por pensamentos autoderrotistas e uma visão pessimista e negativa diante do futuro, e está fortemente relacionada à depressão (Beck, Rush, Shaw \& Emery, 1997). Indivíduos com transtornos depressivos tendem a avaliar negativamente a si mesmos, o mundo e o futuro.

Pode-se dizer que a desesperança está relacionada ao sentimento de fracasso, considerando-se que na velhice o fracasso ocorre com maior freqüência do que na juventude (Skinner \& Vaughan, 1985). Muitas vezes, o idoso deixa de fazer aquilo que não consegue realizar com sucesso, visto que considera que irá fracassar novamente. Nesse momento o sentimento de desesperança é inevitável. Sob esse aspecto, Davim e cols. (2004) preconizam que, com o avançar da idade, existe uma progressiva perda de recursos físicos, mentais e sociais, que conduzem a sentimentos de desamparo. A velhice, muitas vezes, caracteriza-se por sentimentos de impotência e fragilidade.

Considerando-se os aspectos aqui abordados, pode-se aventar a hipótese de que sentimentos característicos do envelhecer podem despertar a desesperança e conduzir a sintomas depressivos e ansiosos. Assim, o objetivo do presente estudo foi explorar a relação entre ansiedade, depressão e desesperança em idosos, bem como buscar eventuais diferenças entre os grupos de asilares e não asilares.

\section{MÉTODO}

\section{Participantes}

Participaram 79 idosos do Sul do Estado de Minas Gerais. Parte deles era proveniente de um centro de terceira idade $(50,6 \% ; n=40)$, que constituiu o grupo 1 , caracterizado por apresentarem seus integrantes melhor situação financeira, podendo ser considerados de classe média e média alta. O segundo grupo foi formado por pessoas freqüentadoras de um posto de retirada de medicamentos ( $38 \% ; n=30)$, integrado por pessoas com situação financeira de classe média baixa. $\mathrm{O}$ terceiro grupo era proveniente de uma instituição asilar $(11,4 \%$; $n=9)$ e composto por idosos de classe média e classe média baixa. A média de idade foi de 69 anos e oito meses $(D P=6,7)$ e a idade máxima constatada neste estudo foi de 84 anos. O gênero feminino representou $79,7 \%(n=63)$ da amostra e o masculino 20,3\% (n=16).

\section{Instrumentos}

Como instrumentos foram utilizados um questionário sociodemográfico, formulado com questões de caracterização dos sujeitos - tais como nome, idade, sexo, estado civil, escolaridade, composição familiar, atividades físicas e sociais e saúde - e Escalas de Beck- versão traduzida e validada para a realidade brasileira (Cunha, 2001) com permissão de The Psychological Corporation, U.S.A., por Jurema Alcides Cunha. Estas consistem de quatro escalas que mensuram sintomas de ansiedade, depressão, desesperança e ideação suicida (nesta pesquisa não se trabalhou com ideação suicida). Cabe destacar que o instrumento apresenta evidências de validade para a população adulta brasileira.

O inventário de ansiedade Beck é composto por 21 itens, com alternativas de respostas variando de nada a um pouco, moderadamente e gravemente. A classificação recomendada para o nível de ansiedade é ansiedade mínima (0-7), ansiedade leve (8-15), ansiedade moderada (16-25) e ansiedade grave (26-63).

O inventário de depressão Beck é composto por 21 itens, cada qual com quatro categorias de respostas. A classificação da intensidade da depressão varia entre depressão mínima (0-9), depressão leve (10-16), depressão moderada (17-29) e depressão severa (30-63).

A escala de desesperança Beck apresenta 20 frases, nas quais o sujeito deve assinalar verdadeiro ou falso para cada afirmação apresentada. A classificação crítica de pessimismo, o que indica a desesperança, varia de nível mínimo de desesperança (0-3) a nível leve de desesperança (4-8), nível moderado de desesperança (9-14) e nível grave (superior a 14).

\section{Procedimento}

Antes da aplicação nos três grupos de idosos estabeleceu-se um bom rapport entre as aplicadoras e os participantes. As escalas foram aplicadas coletivamente em uma única sessão na população que freqüentava o centro de terceira idade. A aplicação ocorreu no próprio centro e teve duração de aproximadamente 50 minutos.

No caso dos idosos que se dirigiam ao posto de retirada de medicamentos, a aplicação foi feita individualmente em um local reservado no posto, tendo duração de aproximadamente 40 minutos cada aplicação. Quando os idosos chegavam ao local, eram abordados e convidados a participar da pesquisa. Foi necessária uma semana para que a coleta se realizasse nesse contexto.

Nos idosos asilares a aplicação também foi individual, tendo duração de aproximadamente 40 
minutos cada uma. Foram necessários dois dias para a realização da coleta na instituição.

Cabe destacar que as aplicadoras permaneceram junto aos idosos para qualquer tipo de auxílio na execução da tarefa. Contudo, observou-se que a compreensão dos itens foi similar nos três grupos, não havendo dificuldades para responderem aos instrumentos. É importante ressaltar que somente os idosos que assinaram o termo de consentimento livre e esclarecido participaram da pesquisa e que todos os procedimentos obedeceram ao que preconiza a Resolução 196/96 do Conselho Nacional de Saúde (Brasil, 1996)

\section{RESULTADOS}

Os dados foram organizados em planilhas e submetidos à estatística descritiva e inferencial, conforme o objetivo do estudo. No que concerne aos dados relativos ao estado civil e moradia, $17,7 \%$ $(n=14)$ são solteiros, sendo que, desse total, 3,8\% ( $n=3)$ são asilares; 32,9\% $(n=26)$ são casados, 35,4\% $(n=28)$ são viúvos e desse percentual 7,6\% (n=6) são asilares; e 13,9\% ( $n=11)$ são separados ou desquitados. A distribuição das respostas dos grupos de idosos do centro de terceira idade e do posto de retirada de medicamentos foi proporcionalmente equilibrada nas categorias.

Quanto à moradia, 32,9\% $(n=26)$ moram sozinhos, $22,8 \%(n=18)$ moram na casa dos filhos, 21,5\% $(n=17)$ moram com o cônjuge e sem parentes ou familiares e apenas $1,3 \%(n=1)$ afirmou morar com um cuidador. Os demais responderam que moram com outros parentes $(n=17 ; 21,5 \%)$. Não houve diferenças entre as respostas dos grupos de idosos do centro de terceira idade e do posto de retirada de medicamentos.

Conforme as respostas a uma questão sobre a fonte de renda do idoso, 74,7\% $(n=59)$ da amostra vivem de aposentadoria, e desse total, 7,6\% (n=6) são asilares; $26,6 \%(n=21)$ vivem de pensão, sendo que 3,8\% (n=3) dos asilares também assinalaram essa modalidade de renda e apenas $5,1 \% \quad(n=4)$ dependem de ajuda de filhos ou familiares. Cabe ressaltar que nessa alternativa o idoso poderia assinalar mais de uma alternativa de resposta. Também se observou que não houve diferença na distribuição das respostas entre os grupos de idosos do centro de terceira idade e do posto de retirada de medicamentos.

Uma questão abordava o nível de instrução escolar dos participantes. A Tabela 1 mostra a distribuição dos participantes considerando a escolaridade, bem como a distribuição da escolaridade por grupo de idosos.

Tabela 1. Frequiência e Porcentagem dos Participantes Considerando a Instrução Escolar, Bem Como os Grupos aos Quais Pertenciam

\begin{tabular}{|c|c|c|c|c|}
\hline Instrução Escolar & $\begin{array}{r}F \text { e } \% \\
\text { Total } \\
\end{array}$ & $\begin{array}{c}F \text { e } \% \\
\text { Centro Terceira Idade }\end{array}$ & $\begin{array}{c}F \text { e } \% \\
\text { Posto Retirada }\end{array}$ & $\begin{array}{c}F \text { e \% } \\
\text { Instituição Asilar }\end{array}$ \\
\hline \multirow[t]{2}{*}{ Ensino fundamental incompleto } & 20 & 13 & 5 & 2 \\
\hline & 25,3 & 32,5 & 16,7 & 22,2 \\
\hline \multirow[t]{2}{*}{ Não possui instrução, mas sabe ler e escrever } & 17 & 12 & 2 & 3 \\
\hline & 21,5 & 30 & 6,7 & 33,3 \\
\hline \multirow{2}{*}{ Ensino fundamental completo } & 15 & 5 & 9 & 1 \\
\hline & 19,0 & 12,5 & 30 & 11,1 \\
\hline \multirow[t]{2}{*}{ Não possui instrução e não sabe ler e escrever } & 8 & 2 & 3 & 3 \\
\hline & 10,1 & 5 & 10 & 33,3 \\
\hline \multirow[t]{2}{*}{ Ensino médio completo } & 6 & 3 & 3 & 0 \\
\hline & 7,6 & 7,5 & 10 & 0 \\
\hline \multirow[t]{2}{*}{ Ensino superior completo } & 5 & 1 & 4 & 0 \\
\hline & 6,3 & 2,5 & 13,3 & 0 \\
\hline \multirow[t]{2}{*}{ Ensino médio incompleto } & 4 & 2 & 2 & 0 \\
\hline & 5,1 & 5 & 6,7 & 0 \\
\hline \multirow[t]{2}{*}{ Ensino médio profissionalizante } & 3 & 2 & 1 & 0 \\
\hline & 3,8 & 5 & 3,3 & 0 \\
\hline \multirow[t]{2}{*}{ Ensino superior incompleto } & 1 & 0 & 1 & 0 \\
\hline & 1,3 & 0 & 3,3 & 0 \\
\hline \multirow[t]{2}{*}{ Total } & 79 & 40 & 30 & 9 \\
\hline & 100 & 100 & 100 & 100 \\
\hline
\end{tabular}

Observa-se que a maior parte dos idosos possui ensino fundamental incompleto e um número considerável, embora não apresente nenhuma instrução escolar, sabe ler e escrever.
Quanto questionados sobre a prática de atividades físicas, a caminhada apareceu com maior frequiência entre os idosos $(44,3 \% ; n=35)$. A caminhada também apareceu como a única atividade física praticada pelo 
asilares, representando 3,8\% ( $n=3)$ da amostra; $29,1 \%$ $(n=23)$ fazem ginástica, a natação é uma atividade realizada por $11,4 \%(n=9), 29,1 \%(n=23)$ se exercitam por meio da dança e 3,8\% (n=3) afirmaram que correm com frequiência. Observa-se que o idoso poderia assinalar mais de uma alternativa de resposta nessa questão e que não houve disparidade nas respostas entre os grupos de idosos do centro de terceira idade e do posto de retirada de medicamentos.

A participação em eventos sociais é realizada com alta freqüência por grande parte dos idosos $(41,8 \% ; n=33)$; uma outra parcela considerável afirma que raramente participa $(26,6 \%, n=21)$, e no caso dos asilares, $2(2,5 \%)$ idosos assinalaram essa questão. Apenas uma pessoa $(1,3 \%)$ afirma que quase sempre participa, pois este não é um comportamento freqüente; e a não-participação em eventos sociais foi apontada por $24,1 \% \quad(n=19)$, sendo que $8,9 \%(n=7)$ dos respondentes constituíam o grupo de asilares. Os demais $(6,3 \% ; n=5)$ não responderam a questão. A equivalência na distribuição das respostas dos grupos de idosos do centro de terceira idade e do posto de retirada de medicamentos foi novamente observada.

Quanto à satisfação com a saúde, $34,2 \%(n=27)$ dizem se sentir "bem", 25,3\% $(n=20)$ afirmam se sentir "ótimo", ressaltando-se que desse total 7,6\% $(n=6) \quad$ são asilares; $30,4 \% \quad(n=24) \quad$ se sentem "regular" e desse montante 3,8\% (n=3) são asilares; e 6,3\% (n=5) relatam sentir-se "péssimo". Algumas idosos $(3,8 \% ; n=3)$ não responderam como se sentem e a categoria "péssimo" foi assinalada por 2 $(n=2,5)$ idosos do centro de terceira idade e por 1 $(n=12,3)$ idoso do posto de retirada de medicamentos.

As dificuldades apresentadas pelos idosos em relação à saúde também foram levantadas. Na Tabela 2 foram elencadas as dificuldades mais freqüentes entre os idosos, salientando-se que o idoso poderia assinalar mais de uma alternativa de resposta para a questão.

Tabela 2. Distribuição da Frequiência e Porcentagem das Dificuldades em Relação à Saúde Apresentadas Pelos $\operatorname{Idosos}(N=79)$

\begin{tabular}{lcc}
\hline Dificuldades em relação à saúde & $\boldsymbol{F}$ & \% \\
\hline Problemas de Pressão & 46 & 58,2 \\
Problemas cardíacos & 14 & 17,7 \\
Problemas cirulatórios & 12 & 15,2 \\
Problemas renais & 8 & 10,1 \\
Diabetes & 7 & 8,9 \\
Problemas respiratórios & 6 & 7,6 \\
\hline
\end{tabular}

Evidencia-se que a maior parte dos idosos apresenta problemas relacionados à pressão; um número considerável $(19 \% ; n=15)$ afirma que apresenta dificuldades, contudo não classifica quais são elas. Cabe mencionar que dificuldades de pressão e circulação foram as mais mencionadas pelos asilares, sendo que 7,6\% (n=6) afirmaram sofrer esses problemas. Não houve diferença na distribuição das respostas entre os grupos de idosos do centro de terceira idade e do posto de retirada de medicamentos.

As escalas de ansiedade, depressão e desesperança também foram respondidas pelos idosos. A escala de ansiedade foi respondida por $77,2 \%(n=61)$ dos idosos, o inventário de depressão por 73,4\% (n=58) e a escala de desesperança por $81 \%(n=64)$. A Tabela 3 apresenta os dados dos idosos considerando os grupos aos quais pertenciam.

Tabela 3. Distribuição dos Idosos Considerando os Grupos aos Quais Pertenciam

\begin{tabular}{lccc}
\hline Grupos de Idosos & $\begin{array}{c}\boldsymbol{M} \text { e } \boldsymbol{D p} \\
\text { Depressão }\end{array}$ & $\begin{array}{c}\boldsymbol{M} \text { e } \boldsymbol{D p} \\
\text { Ansiedade }\end{array}$ & $\begin{array}{c}\boldsymbol{M} \text { e } \boldsymbol{D} \boldsymbol{p} \\
\text { Desesperança }\end{array}$ \\
\hline Centro da Terceira Idade & 7,8 & 7,7 & 4,1 \\
& 11,2 & 4,8 & 3,6 \\
Posto de Retirada de & 4,8 & 6,4 & 4,9 \\
Medicamentos & 4,8 & 7,8 & 3,7 \\
Instituição Asilar & 13 & 30,2 & 9,1 \\
& 7,7 & 8,9 & 3,2 \\
\hline
\end{tabular}

Para verificar se havia relação entre ansiedade, depressão e desesperança recorreu-se ao teste de correlação de Pearson. A Tabela 4 mostra a distribuição dos índices de correlação entre as variáveis.

Tabela 4. Índices de Correlação Entre Ansiedade, Depressão e Desesperança $(N=79)$

\begin{tabular}{lccc}
\hline Correlações & & Depressão & Desesperança \\
\hline Ansiedade & $r$ & 0,754 & 0,413 \\
& $p$ & 0,000 & 0,002 \\
Depressão & $r$ & & 0,434 \\
& $p$ & & 0,002 \\
\hline
\end{tabular}

Evidencia-se que há relação significativa entre ansiedade, depressão e desesperança entre os idosos pesquisados. Vale ressaltar que o índice de correlação foi expressivamente mais alto entre ansiedade $\mathrm{e}$ depressão.

A análise de variância foi utilizada para verificar possíveis diferenças entre os grupos, considerando-se ansiedade, depressão e desesperança. Os resultados indicaram a existência de diferença estatisticamente significativa entre os grupos no que concerne a ansiedade e desesperança, considerando-se $[F(2,58)=30,35 ; p=0,000]$ e $[F(2,61)=6,38 ; p=0,003]$. 
A variável depressão apresentou diferença marginalmente significativa $[F(2,55)=3,09 ; p=0,053]$.

$\mathrm{O}$ teste $a d-h o c$ de Tukey apontou que na variável ansiedade a diferença estava entre o grupo de idosos proveniente do centro de terceira idade (G1) e o grupo dos idosos que vivem em instituição asilar (G3) $(p<0,001)$. Também houve diferença na pontuação média de ansiedade entre o grupo de idosos do posto de medicamentos (G2) e o grupo de idosos asilares (G3) $(p<0,001)$. Os idosos asilares, nos dois casos, apresentaram um maior nível de ansiedade (escore 30,2 ; ansiedade grave) em relação aos outros dois grupos (escore do $\mathrm{G} 1=7,7$ e do $\mathrm{G} 2=6,4$; ansiedade leve).

A diferença na variável desesperança, segundo o teste ad-hoc de Tukey, ocorreu entre o G1 e G3 $(p=0,002)$ e $\mathrm{G} 2$ e $\mathrm{G} 3(p=0,010)$. Nos dois casos, os idosos asilares obtiveram um maior nível de desesperança (escore 9,1; desesperança moderada) em comparação aos outros dois grupos (escore do $\mathrm{G} 1=4,1$ e do $\mathrm{G} 2=4,9$; desesperança leve). No entanto, a diferença apontada pelo teste ad-hoc de Tukey na variável depressão apareceu entre o G2 e G3 $(p=0,047)$. Os resultados dos idosos do asilo indicaram também níveis mais acentuados de depressão, a saber, depressão leve (escore 13) em relação aos idosos do G2 que apresentou depressão mínima (escore 4,8).

\section{DISCUSSÃO E CONCLUSÃO}

Neste estudo a maior parte dos idosos $(54,4 \%$; $n=43$ ) apresentou independência, visto que eles moram sozinhos ou com o cônjuge e sem parentes ou familiares. As fontes de renda mais comuns são a aposentadoria e a pensão. Também chama a atenção o dado que mostra que a maior parte dos idosos $(68,3 \%$; $n=54$ ) possui alguma instrução escolar.

Sob esse aspecto, Birren e Schroots (2001) defendem ser comum que muitos idosos continuem a levar uma vida saudável e com um bom grau de independência, principalmente aqueles que não apresentam nenhuma debilidade grave em relação à saúde ou necessidade de ajuda financeira. $\mathrm{O}$ fato de os idosos apresentarem independência em relação a sua vida leva o idoso a encarar de forma natural mais esse processo dinâmico de sua vida (Fraiman, 1995).

A prática de atividades físicas é um hábito de vida saudável em qualquer faixa etária. Na velhice esse hábito promove a manutenção de uma boa saúde física e mental. Os dados apontaram que a realização de atividades físicas foi comum entre os idosos, sendo que a caminhada foi a atividade mais freqüente nessa população. A participação em eventos sociais também pode ser observada com alguma freqüência em uma boa parcela dos idosos $(41,8 \% ; n=33)$, contudo uma parte dos idosos $(24,1 \% ; n=19)$ afirmou não participar de nenhum evento social.

Uma velhice vivenciada de forma positiva, para Bee e Mitchell (1984), envolve a prática de atividades físicas e de lazer, o que certamente promove a melhora da qualidade de vida. Pessoas que se sentem bem e felizes nessa faixa apresentam bons relacionamentos sociais e emoções que geram prazer e realização. Desse modo, sugere-se que os participantes, de uma forma geral, comprovaram uma vivência positiva em relação a sua velhice, apresentando inclusive hábitos saudáveis que corroboram a tranqüilidade para uma boa saúde mental.

A satisfação percebida pelos idosos em relação à saúde foi apontada como boa e ótima pela maior parte dos idosos. Todavia, chama a atenção o fato de que cinco idosos relataram sentir-se péssimos quanto a sua saúde. Nessa direção, Fleck e cols. (2002) consideram que pessoas com maior intensidade de sintomas depressivos não estão satisfeitas com sua saúde. Pessoas com sintomas depressivos tendem a apresentar distúrbios ansiosos e, consequientemente, algum nível de desesperança (Gazalle, Lima, Tavares \& Hallal, 2004; Solomon, 2002).

Nesse sentido, constatou-se relação entre ansiedade, depressão e desesperança, sendo que o índice de correlação foi mais alto entre ansiedade e depressão. As pessoas que apresentaram ansiedade, também apresentaram depressão e desesperança em alguma intensidade. Esses dados não causam estranheza, visto que já foram observados anteriormente em pesquisas como a de Beck e cols. (1997), que mostrou estar a desesperança relacionada à depressão, a de Veras e Coutinho (1991) e a de Xavier e cols. (2001) e Byrne (2002), que associaram a ansiedade com sintomas depressivos.

A diferença entre os grupos quanto aos sintomas de ansiedade, depressão e desesperança também foi constada. Em todas as variáveis o grupo de idosos asilares apresentou uma maior incidência de sintomas. $\mathrm{O}$ caso mais preocupante foi a incidência de ansiedade nessa população, apresentando uma classificação considerada grave, embora nos outros dois grupos o nível de sintomas ansiosos tenha sido considerado leve.

A desesperança também foi constatada em nível moderado nessa população, sendo que nos outros dois grupos foi observado um nível leve de desesperança. A depressão apresentou diferença entre os grupos de idosos que retiram remédios de um posto de 
distribuição e os idosos asilares. Os idosos asilares apresentaram uma maior incidência de sintomas depressivos, classificados como depressão leve. Chama a atenção o dado de que os idosos que frequientam o centro de terceira idade também apresentaram depressão mínima, assim como os idosos do grupo dos que retiram remédios de um posto de distribuição.

Corroborando esses resultados, a pesquisa de Davim e cols. (2004) com idosos que viviam em instituições asilares indicou que idosos que moram nessas instituições estão mais propensos a apresentar dificuldades relacionadas à saúde física e psicológica. Os dados desta pesquisa caminham na direção dos estudos de Davim e cols. (2004), ao passo que os idosos asilares apresentam mais sintomas de ansiedade, depressão e desesperança.

Considerando-se as três variáveis, a ansiedade apresentou uma incidência de sintomas mais grave nessa população. Esse fato talvez esteja relacionado a uma visão catastrófica do idoso em relação a diversas situações de sua vida, o que gera o sentimento de que algo perigoso e ameaçador pode acontecer (Skinner \& Vaughan, 1985). Faltam ao idoso asilar perspectivas positivas em relação a sua própria vida. $\mathrm{O}$ sentimento de abandono e instabilidade em relação ao futuro é constante, daí o aparecimento de sintomas ansiosos.

\section{REFERÊNCIAS}

Albuquerque, S. R. T. P. (1994). Viabilidade de um centro para terceira idade. Psicologia Argumento, 12(14), 83-100.

Almeida, O. P. (1999). Idosos atendidos em serviço de emergência de saúde mental: características demográficas e clínicas. Revista Brasileira de Psiquiatria, 21(1), 12-18.

American Psychiatric Association (1994). Manual de diagnóstico e estatística dos distúrbios mentais (DSM-IV). Porto Alegre: Artes Médicas.

Antonucci, T. C. (2001, $4^{\text {th }}$ ed.). Social relations: An examination of social networks, social support, and sense of control. In J. E. Birren \& K. W. Schaie (Eds.), Handbook of the psychology of aging. (pp. 427-453). California: Academic.

Beck. A T., Rush A J., Shaw, B. F. \& Emery, G. (1997). Terapia cognitiva da depressão. (Sandra Costa, Trad.). Porto Alegre: Artes Médicas.

Bee, H. L. \& Mitchell, S. K. (1984). A pessoa em desenvolvimento. São Paulo: Editora Harper \& Row do Brasil.

Birren, J. E. \& Schroots, J. J. F. (2001, $4^{\text {th }}$ ed.). History, concepts and theory in the psychology of aging. In J. E. Birren \& K. W. Schaie (Eds.), Handbook of the psychology of aging. (pp. 03-23). California: Academic.
Byrne, G. (2002). What happens to anxiety disorders in later life? Revista Brasileira de Psiquiatria, 24(1), 74-80.

Coes, M. C. R. (1991). Ansiedade: uma avaliação quantitativa de seus efeitos negativos sobre o desempenho no vestibular. Psicologia: Teoria e Pesquisa, 7(2), 137-147.

Brasil (1996). Conselho Nacional de Saúde. Resolução 196/96. Diretrizes e Normas Regulamentadoras de Pesquisa. Disponível em: <http://www.conselho.saude.gov.br>. (Acesso em 19/11/2005).

Cunha, J. (2001). Manual em português das Escalas Beck. São Paulo: Casa do Psicólogo.

Dalgalarrondo, P. (2000). Psicopatologia e semiologia dos transtornos mentais. Porto Alegre: Artes Médicas.

Davim, R. M. B., Torres, G. V., Dantas, S. M. M. \& Lima, V. M. (2004). Estudo com idosos de instituições asilares no município de Natal/RN: características socioeconômicas e de saúde. Revista Latino Americana de Enfermagem, 12(3), 518-524.

Fleck, M. P. A., Lima, A. F. B. S., Louzada, S., Schestasky, G., Henriques, A., Borges, V. R. \& Camey, S. (2002). Associação entre sintomas depressivos e funcionamento social em cuidados primários à saúde. Revista de Saúde Pública, 36(4), 431-438.

Fraiman, A. P. (1995, $3^{\text {a }}$ ed.). Coisas da idade. São Paulo: Editora Gente.

Garbin, T. R. \& Montewka, A. (2000). Análise de condições ambientais: um estudo sobre variáveis que facilitam a autonomia do idoso institucionalizado. Em Livro de resumos IX Encontro Brasileiro de Psicoterapia e Medicina Comportamental. (pp. 13-14). Campinas.

Gazalle, F. K., Hallal, P. C. \& Lima, M. S. (2004). Depressão na população idosa: os médicos estão investigando? Revista Brasileira de Psiquiatria, 26(3), 145-149.

Gazalle, F. K., Lima, M. S, Tavares, B. F. \& Hallal, P. C. (2004). Sintomas depressivos e fatores associados em uma população idosa no sul do Brasil. Revista de Saúde Pública, 38(3), 365-71.

Haddad, E. G. M. (1986). A ideologia da velhice. São Paulo: Cortez.

Haddad, E. G. M. (1993). A velhice em movimento. Gerontologia, 1(1), 29-30.

Katona, C., Manela, M. \& Livingston, G. (1996). How common are the anxiety disorders in old age? Geriatric Psychiatry, (11), 65-70.

Lawton, M. P. (2001, $4^{\text {th }}$ ed.). Quality of life and the end of life. In J. E. Birren \& K. W. Schaie (Eds.), Handbook of the psychology of aging. (pp. 592-616). California: Academic.

Maia, L. C., Durante, A. M. G. \& Ramos, L. R. (2004). Prevalência de transtornos mentais em área urbana no norte de Minas Gerais. Revista de Saúde Pública, 38(5), 650-656.

Neri, A. L. \& Nogueira, E. J. (1994). Como a velhice é apresentada às crianças em textos de literatura infantil brasileira. Pro-Posições, 5(1), 45-71. 
Neri, A. L. (1995). Psicologia do envelhecimento: uma área emergente. Em A. L. Néri (Org.), Psicologia do envelhecimento: temas selecionados na perspectiva de curso de vida. (pp. 13-40). Campinas: Papirus.

Piccoloto, N., Wainer, R., Benvegnú, L. \& Juruena, M. (2001). Revisão comparativa entre os transtornos do humor. Revista de Psiquiatria Clínica, 27(2). Disponível em: <http://www.www.hcnet.usp.br/ipq/revista>. (Acesso em 21/07/2004).

Regier, D. A., Boyd, J. H., Burke, J. D., Rae, D. S., Myers, J. M., Krammer, M., Robins, L. K., George, M. K. \& Locke, B. Z. (1988). One-month prevalence of mental disorders in the United States: Based on five epidemiologic catchments area sites. Arch Gen Psychiatry, 45, 977-986.

Santos, M. F. S. (1994). Velhice: uma questão psico-social. Temas em Psicologia, 2, 123-131.

Skinner, B. F. \& Vaughan, M. E. (1985). Viva bem a velhice: aprendendo a programar a sua vida. (A. L. Neri, Trad.). São Paulo: Summus.
Solomon, A. (2002). O Demônio do meio-dia: uma anatomia da depressão. Rio de Janeiro: Objetiva.

Veras, R. P. \& Coutinho, E. S. F. (1991). Estudo de prevalência de depressão e síndrome cerebral orgânica na população de idosos. Revista de Saúde Pública, 25(3), 209-217.

Xavier, M. F. F., Ferraz, M. P. T., Trendi, C. M., Argimon, I., Bertollucci, P.H., Poyares, D. \& Moriguchi, E. H. (2001). Transtorno de ansiedade generalizada em idosos com 80 anos ou mais. Revista de Saúde Pública, 35(3), 294-302.

Recebido em 14/12/2005 Aceito em 09/03/2006

Endereço para correspondência: Katya Luciane de Oliveira. R. Maestro Sebastião Peranovich, 415, B. Atibaia Jardim, CEP 12940-000, Atibaia-SP. E-mail: katya_lincoln@ig.com.br 